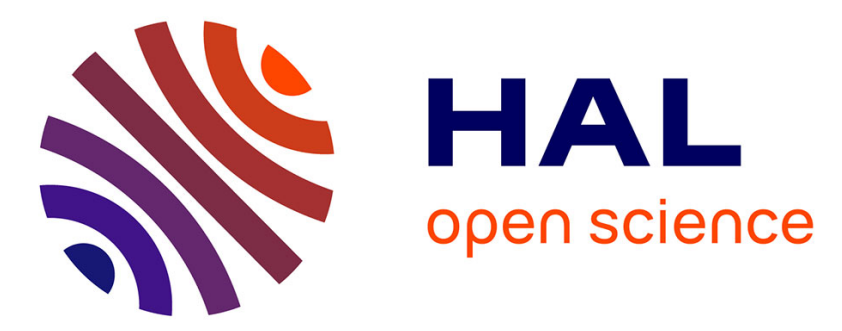

\title{
A piecewise-polynomial approach to the stability analysis of non-linear switching controllers in presence of sliding modes with application to pneumatic systems
}

\author{
Omar Ameur, Paolo Massioni, Gérard Scorletti, Xavier Brun, Mohamed
} Smaoui

\section{To cite this version:}

Omar Ameur, Paolo Massioni, Gérard Scorletti, Xavier Brun, Mohamed Smaoui. A piecewisepolynomial approach to the stability analysis of non-linear switching controllers in presence of sliding modes with application to pneumatic systems. 54th IEEE CDC, Dec 2015, Osaka, Japan. pp.48724878, 10.1109/CDC.2015.7402980 . hal-01246952

\section{HAL Id: hal-01246952 \\ https://hal.science/hal-01246952}

Submitted on 30 Apr 2019

HAL is a multi-disciplinary open access archive for the deposit and dissemination of scientific research documents, whether they are published or not. The documents may come from teaching and research institutions in France or abroad, or from public or private research centers.
L'archive ouverte pluridisciplinaire HAL, est destinée au dépôt et à la diffusion de documents scientifiques de niveau recherche, publiés ou non, émanant des établissements d'enseignement et de recherche français ou étrangers, des laboratoires publics ou privés. 


\title{
A piecewise-polynomial approach to the stability analysis of non-linear switching controllers in presence of sliding modes with application to pneumatic systems
}

\author{
Omar Ameur, Paolo Massioni, Gérard Scorletti, Xavier Brun and Mohamed Smaoui
}

\begin{abstract}
This paper concerns the stability analysis of nonlinear, switching control laws for pneumatic actuators. A first approach to the problem has been proposed by the authors in [1], under the hypothesis of a simplified model of friction. The approach is based on casting the closed-loop system into a piecewise-affine form. However, if a more realistic friction model is introduced, the method in [1] proves to be too conservative, and unfit to deal with the sliding modes that can occur with this new model. This paper proposes a new method for proving the stability on the system by introducing a less conservative class of Lyapunov function, namely piecewisepolynomial ones. At the end of the paper, we show how such a method can be successfully applied to our experimental setup.
\end{abstract}

\section{INTRODUCTION}

Friction is a complex phenomenon which plays a key role in the dynamic behavior of several industrial systems. For example, in pneumatic cylinders, the presence of the friction may lead to what is called "stick-slip" [17], [5], a phenomenon consisting in jerky mouvements of the rod after it has come to a rest; such a behaviour is highly undesirable in the industrial applications and it can even lead to limit cycles.

A control law which avoids the occurrence of stick-slip has been proposed recently in [27]. This solution consists in a classic feedback linearization, which accounts for the nonlinearities in the pneumatic model, together with an appropriate switching law. The controller switches from a position tracking control to a pressure control after the rod has come to a rest, in order to reduce the pressure difference between the two chambers, avoiding an uncontrolled evolution that eventually could make the rod restart. A major problem with this solution, which had been otherwise verified as very effective in the practice, was to find a formal proof of its stability. So a first approach to the problem has been given in [1], where the stability analysis of this system with the switched controller has been takled by casting the closed-loop system into a piecewise-affine (PWA) form [9], [21], [24], [19]. For this class of systems, new sufficient conditions for stability have been proposed based on piecewise-quadratic Lyapunov functions and given in terms of linear matrix inequalities (LMIs).

O. Ameur and G. Scorletti are with Laboratoire Ampère, UMR CNRS 5005, École Centrale de Lyon, Université de Lyon, \{omar.ameur, gerard.scorletti\}@ec-lyon.fr

P. Massioni, X. Brun and M. Smaoui are with Laboratoire Ampère, UMR CNRS 5005, INSA de Lyon, Université de Lyon, \{paolo.massioni, xavier.brun, mohamed.smaoui\}einsa-lyon.fr
In [1], the authors have applied the piecewise-affine approach to prove the stability of the switching non-linear control laws for pneumatic actuators; nevertheless, this proof is valid under a simplifying hypothesis for the model of the friction. In fact, the friction has been described as a continuous function of the velocity of the rod (a so-called "saturation" shape); however, as indicated by many works [2], [8], [28], [6], more accurate models of friction feature discontinuities, which can lead to the appearance of sliding modes [11], [10] in the system dynamics. The approach proposed in [1] is not able to deal with discontinuities and sliding modes, so no conclusion on the stability can be drawn with this approach if the realistic model of friction is taken into account. So, the research work presented in this paper aims at giving an answer to this problem, i.e. find a theoretical tool able to prove the stability of the switching law applied to the cylinder even in the presence of sliding modes.

The literature already contains several approaches for dealing with sliding modes in piecewise-affine systems [15], [9], [4], [13], [25]. In particular [15] presents an extension of the well-known methods presented in [16]. In [9], these former works has been extended in order to cope with the presence of sliding mode even in the case of an equilibrium point belonging to the boundary between two cells. The conditions in [9] combine the work of [25] and the work of [22] on the stabilization of piecewise affine systems in the presence of sliding modes. However, these method are still too conservative for our specific problem, as they rely on combinations of piecewise-quadratic Lyapunov functions. In [4] the sliding modes have been considered as a system dynamics; the drawback in this case is the need for more information on the sliding modes. This drawback has been avoided in the work of [25] but conservatively using common Lyapunov functions. Alas, as we will see, all of these approaches are not enough to deal with the stability of our pneumatic actuator systems. First of all, we have verified that searching for a piecewise-quadratic Lyapunov functions is too conservative and does not yield any valid solution. Secondly, these former methods are not able to cope with the presence of a whole equilibrium set (not only a point) in a sliding mode. This paper shows how we extend the previous works, introducing piecewise-polynomial Lyapunov functions.

The paper is organized as follows. Section II contains the description of the pneumatic actuator model with the 
proposed switching control law. Section III introduces the PWA class of systems and the tools that can be used for the stability analysis in the presence of sliding modes. Section IV contains the main theoretical result, i.e. a method for proving stability, whereas Section V shows its application to our test bench model. The conclusions are given in Section VI.

\section{PNEUMATIC ACTUATOR SYSTEMS}

\begin{tabular}{|c|c|}
\hline$y$ & position of the piston rod (m) \\
\hline$v$ & velocity $\left(\mathrm{m} \mathrm{s}^{-1}\right)$ \\
\hline$p_{P}, p_{N}$ & pressures in the cylinder chambers $(\mathrm{Pa})$ \\
\hline & acceleration $\left(\mathrm{m} \mathrm{s}^{-2}\right)$ \\
\hline$F_{s}$ & stiction friction force $(\mathrm{N})$ \\
\hline$k$ & gas polytropic constant \\
\hline$l$ & length of stroke $(\mathrm{m})$ \\
\hline$M$ & moving load (carriage and rod mass) $(\mathrm{kg})$ \\
\hline$q_{m P}, q_{m N}$ & $\begin{array}{l}\text { mass flow rate provided by the servovalves } \\
\text { to the cylinder chambers }\left(\mathrm{kg} \mathrm{s}^{-1}\right)\end{array}$ \\
\hline$r$ & perfect gas constant $\left(\mathrm{J} \mathrm{kg}^{-1} \mathrm{~K}^{-1}\right)$ \\
\hline$S$ & area of cylinder bore $\left(\mathrm{m}^{2}\right)$ \\
\hline$T$ & temperature $(\mathrm{K})$ \\
\hline$V$ & volume $\left(\mathrm{m}^{3}\right)$ \\
\hline$V_{D}$ & dead volume of cylinder chamber $\left(\mathrm{m}^{3}\right)$ \\
\hline$b_{v}$ & viscous friction coefficient $\left(\mathrm{N} \mathrm{m}^{-1} \mathrm{~s}^{-1}\right)$ \\
\hline
\end{tabular}

The pneumatic system that we consider is shown in Fig. 1. It comprises an actuator in the form of a pneumatic cylinder (double acting) with a rod connected to a carriage on rails. The actuator is powered by compressed air, with two servovalves for controlling the flow supplied to both the chambers of the cylinder. Two sensors measure the pressures in the chambers.

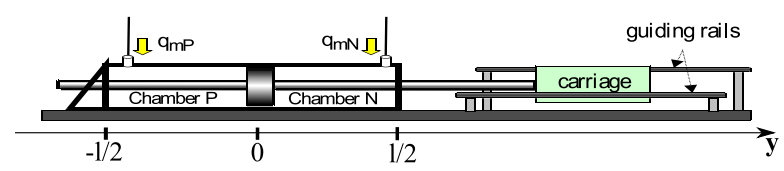

Fig. 1. Electro-pneumatic actuator.

Based on [1], [27], the physical model of the system in open loop is given by the following equations:

$$
\left\{\begin{array}{l}
\dot{y}=v \\
\dot{v}=\frac{1}{M}\left(S\left(p_{P}-p_{N}\right)-b_{v} v-F_{f}(v)\right) \\
\dot{p}_{N}=\frac{k r T}{V_{N}(y)}\left(\frac{S}{r T} p_{N} v+q_{m N}\right) \\
\dot{p}_{P}=\frac{k r T}{V_{P}(y)}\left(\frac{-S}{r T} p_{P} v+q_{m P}\right)
\end{array}\right.
$$

where (see Table I for the notation) the inputs are the two mass flow rates $q_{m P}$ and $q_{m N}$, and $V_{P}(y)=V_{0}+S y$, $V_{N}(y)=V_{0}-S y$ with $V_{0}=V_{D}+S \frac{l}{2}$. The friction force $F_{f}(v)$ is described by the following discontinuous, "relay" model:

$$
F_{f}(v) \begin{cases}=+F_{s} & \text { for } v>0 \\ \in\left[-F_{s},+F_{s}\right] & \text { for } v=0 \\ =-F_{s} & \text { for } v<0\end{cases}
$$

Notice the difference with respect to the continuous ("saturation") model chosen in [1]. The switching control law proposed in [1], [27] is based on a feedback linearization of the model in (1), which cancels out all the nonlinearities of the system but the ones caused by friction (as we assume uncertainties in this model, so exact cancellation is impossible). Then, the controller switches between the two following laws.

\section{- Position tracking law (\#1)}

$$
\left\{\begin{array}{r}
q_{m P}=\frac{V_{P}(y)}{k r T}\left[\frac{k S}{V_{P}(y)} v p_{P}+\dot{p}_{P d}-k_{P} e_{P}\right] \\
q_{m N}=\frac{M V_{N}(y)}{S k r T}\left[\frac{S k r T}{M V_{P}(y)} q_{m P}+\frac{S^{2} k v}{M}\left(-\frac{p_{P}}{V_{P}(y)}-\frac{p_{N}}{V_{N}(y)}\right)\right. \\
\left.\quad-\frac{b_{v}}{M} a-j_{d}+k_{a} e_{a}+k_{v} e_{v}+k_{y} e_{y}\right]
\end{array}\right.
$$

which allows the tracking of a given time-varying position reference. It results in the following closedloop dynamics:

$$
\left\{\begin{array}{l}
\dot{e}_{y}=e_{v} \\
\dot{e}_{v}=e_{a f}-\frac{1}{M} F_{f}(v) \\
\dot{e}_{a f}=-k_{a} e_{a f}-k_{v} e_{v}-k_{y} e_{y}+\frac{k_{a}}{M} F_{f}(v) \\
\dot{e}_{P}=-k_{P} e_{P}
\end{array}\right.
$$

\section{- Pressure control law (\#2)}

$$
\left\{\begin{array}{l}
q_{m P}=\frac{V_{P}(y)}{k r T}\left[\frac{k S}{V_{P}(y)} v p_{P}+\dot{p}_{P d}-k_{P} e_{P}\right] \\
q_{m N}=\frac{V_{N}(y)}{k r T}\left[-\frac{k S}{V_{N}(y)} v p_{N}+\dot{p}_{N d}-k_{N} e_{N}\right]
\end{array}\right.
$$

which regulated the pressures in the two chambers in order to avoid stick-slip; this law is active when the carriage has arrived at the desired position. The resulting closed-loop dynamics is

$$
\left\{\begin{array}{l}
\dot{e}_{y}=e_{v} \\
\dot{e}_{v}=e_{a f}-\frac{1}{M} F_{f}(v) \\
\dot{e}_{a f}=\frac{S}{M}\left(k_{N}-k_{P}\right) e_{P}-k_{N} e_{a f} \\
\dot{e}_{P}=-k_{P} e_{P}
\end{array}\right.
$$

In all of these equations above, $e_{a f}=e_{a}+\frac{1}{M} F_{f}(v)$. $e_{y}, e_{v}, e_{a}, e_{P}, e_{N}$ are the errors between states and their desired values; the constants $k_{y}, k_{v}, k_{a}, k_{P}$ and $k_{N}$ are the state feedback gains chosen by a pole-placement on the feedback-linearized model. The switching criterion is

$$
\begin{aligned}
& \# 1 \rightarrow \# 2: v_{d}=0 \wedge\left|e_{y}\right| \leq \varepsilon_{1} \wedge\left|e_{v}\right| \leq \varepsilon_{2} \\
& \# 2 \rightarrow \# 1: v_{d} \neq 0 \vee\left|e_{y}\right|>\varepsilon_{1} \vee\left|e_{v}\right|>\varepsilon_{2}
\end{aligned}
$$

where $\varepsilon_{1}$ and $\varepsilon_{2}$ are small arbitrary constants. The above switching controller has shown no instability both in simulation and on the test bench and has never caused the occurrence of stick-slip (as shown in Fig. 2). The topic of the rest of this paper is the formal proof of stability.

\section{PROPOSED APPROACH}

Let us introduce a partition of $\mathbb{R}^{n}$ into $N$ polyhedral cells $X_{i}$ with disjoint interior, with $i \in \mathcal{I}$, a set of $N$ valid indices. We partition $\mathcal{I}=\mathcal{I}_{0} \cup \mathcal{I}_{1}$ (with $\mathcal{I}_{0} \cap \mathcal{I}_{1}=\emptyset$ ) such as $0 \in X_{i}$ 


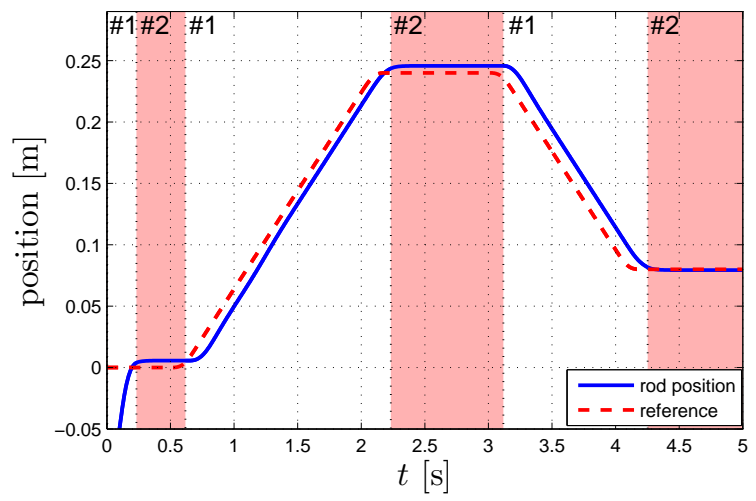

Fig. 2. Evolution of position with respect to the desired position. The background colors are related to the active control mode (\#1 or \#2).

if $i \in \mathcal{I}_{0}$, otherwise $0 \notin X_{i}$ if $i \in \mathcal{I}_{1}$ (the cells with index in $\mathcal{I}_{0}$ contain the origin).

Definition 1: we call a dynamical system "piecewise affine" (PWA) if it has the following dynamics [1], [16], [12]:

$$
\dot{x}(t)=A_{i} x(t)+a_{i} \text { for } x(t) \in X_{i}, i \in \mathcal{I}
$$

where $x(t) \in \mathbb{R}^{n}$ denotes the state-space vector. Equivalently, for $\bar{x}(t)^{T}=\left[x(t)^{T}, 1\right]$

$$
\dot{\bar{x}}(t)=\underbrace{\left[\begin{array}{cc}
A_{i} & a_{i} \\
0 & 0
\end{array}\right]}_{\bar{A}_{i}} \bar{x}(t) \quad \text { for } x(t) \in X_{i}, i \in \mathcal{I}
$$

The domain of each cell $X_{i}$ is described by matrices $\bar{E}_{i} \in$ $\mathbb{R}^{l_{i} \times(n+1)}$ such as:

$$
x \in X_{i} \Rightarrow \bar{E}_{i} \bar{x} \geq 0,
$$

and the boundaries between two cells $X_{i}$ and $X_{j}$ by matrices $\bar{F}_{i j} \in \mathbb{R}^{r_{i j} \times(n+1)}$ such as:

$$
\left\{\begin{array}{l}
\Gamma=\left\{(i, j) \mid X_{i} \cap X_{j} \neq \emptyset\right\} \\
\forall(i, j) \in \Gamma, X_{i} \cap X_{j} \subseteq\left\{x \mid \bar{F}_{i j} \bar{x}=0\right\} .
\end{array}\right.
$$

We then focus on PWA systems satisfying a set of given assumptions.

Assumption 1: on any boundary in $\Gamma$ between two neighboring cells $X_{i}$ and $X_{j}$, a sliding mode may occur (see figure 3), i.e. for $x \in X_{i} \cap X_{j},(i, j) \in \Gamma$ we have the following dynamics (according to Filippov [10]):

$$
\dot{\bar{x}}(t)=\left(\theta \bar{A}_{i}+(1-\theta) \bar{A}_{j}\right) \bar{x}(t), 0 \leq \theta \leq 1 .
$$

Assumption 2: the set $E_{e q} \supseteq\{0\}$ of the equibrium points for (6) is a subset of $\bigcup_{i \in \mathcal{I}_{0}} X_{i}$ (notice that the equilibrium set might also be on a sliding mode).

Assumption 3: we distinguish two cases:

- if the origin is in the interior of a cell $X_{i}$ (for which we have then that $\left.\mathcal{I}_{0}=\{i\}\right): \bar{A}_{i}$ does not have any constant terms $\left(a_{i}=0\right)$; we define $\overline{\mathcal{A}}_{0}=\bar{A}_{i}$;

- if the origin is in the boundary between cells $X_{i}$ and $X_{y}$ : there exists a $\bar{\theta}, 0 \leq \bar{\theta} \leq 1$, for which $\bar{\theta} a_{i}+(1-\bar{\theta}) a_{j}=$ 0 ; we define $\overline{\mathcal{A}}_{0}=\bar{\theta} \bar{A}_{i}+(1-\bar{\theta}) \bar{A}_{j}$.
Moreover, $\overline{\mathcal{A}}_{0}$ may have $h$ eigenvectors (shared with $\bar{A}_{i}$ and $\bar{A}_{j}$ in the second case) that have 0 as associated eigenvalue. We call $Z \in \mathbb{R}^{(n+1) \times h}$ the full-rank matrix of these eigenvectors $\left(\overline{\mathcal{A}}_{0} Z=\bar{A}_{j} Z=\bar{A}_{i} Z=0\right.$ ) (this implies that the equilibrium set can be larger than the origin alone, i.e. $\left.E_{\text {eq }} \supseteq\{0\}\right)$. We then define $\Pi \in \mathbb{R}^{(n+1) \times(n+1-h)}$ as the orthogonal complement of $Z$, normalized such as $\Pi^{T} \Pi=I$.

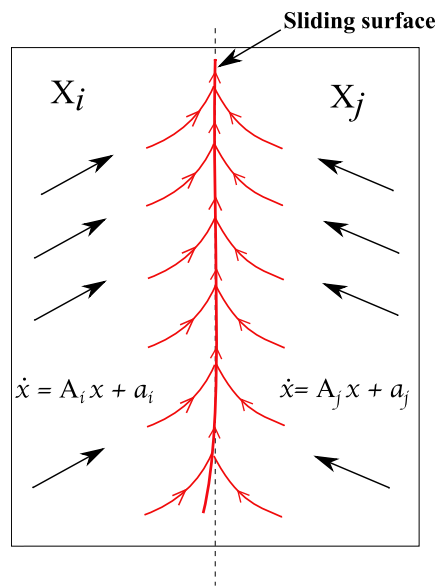

Fig. 3. Sliding modes.

The objective now is to find a set of conditions ensuring the asymptotic convergence of the system state $x(t)$ to the set of equilibrium points $E_{e q}$, notwithstanding the presence of sliding modes on the boundaries. First of all, we have to upgrade the methods in [1] in order to cope with sliding modes. Secondarily, in order to reduce conservatism to the minimum, we need to go beyond the classical piecewisequadratic Lyapunov functions. For this reason, we will make use of Lyapunov function of higher degree, i.e. piecewisepolynomial Lyapunov functions, and given by the expression

$$
V(x)=V_{i}(x) \text { for } x \in X_{i}, i \in \mathcal{I}
$$

such that $V_{i}(x)$ is a polynomial in $n$ variables with degree $2 m$ given by:

$$
V_{i}(x)=\chi(x)^{T} P_{i} \chi(x)
$$

where $P_{i}=P_{i}^{T} \in \mathbb{R}^{\rho \times \rho}$, and $\chi(x) \in \mathbb{R}^{\rho \times 1}$ is the vector of all the monomials of degree less than or equal to $m$ that can be made from the elements of $x ; \rho=\left(\begin{array}{c}m+n \\ m\end{array}\right)$. For example,

$$
\begin{aligned}
\chi(x) & =\left[x_{1}, x_{2}, \ldots, x_{n}, x_{1} x_{2}, x_{1} x_{3}, \ldots, x_{1} x_{n}, x_{2} x_{3}, \ldots\right. \\
& \left.\ldots, x_{2} x_{n}, \ldots, x_{n-1} x_{n}, \ldots, x_{1}^{m}, x_{2}^{m}, \ldots, x_{n}^{m}, 1\right]^{T} .
\end{aligned}
$$

As seen in [23], the choice of $P_{i}$ is not unique for a given polynomial $V_{i}(x)$; there exist a number $\iota$ of linear independent matrices $Q_{\nu}=Q_{\nu}^{T} \in \mathbb{R}^{\rho \times \rho}$ (defined up to a scalar factor), for which

$$
\chi(x)^{T} Q_{\nu} \chi(x)=0 \text { for } \nu=1, \ldots, \iota
$$


with

$$
\iota=\frac{1}{2}\left(\left(\begin{array}{c}
m+n \\
m
\end{array}\right)^{2}+\left(\begin{array}{c}
m+n \\
m
\end{array}\right)\right)-\left(\begin{array}{c}
n+2 m \\
2 m
\end{array}\right)
$$

This implies that $V_{i}(x)=\chi(x)^{T} P_{i} \chi(x)=\chi(x)^{T}\left(P_{i}+\right.$ $\left.\sum_{\nu=1}^{\iota} Q_{\nu} \tau(\nu)\right) \chi(x)$ for any real scalars $\tau(\nu)$. This is due to the fact that some monomials in $V_{i}$ can be obtained as products of different elements in $\chi$, e.g. $x_{1}^{2}=x_{1} \cdot x_{1}=$ $1 \cdot x_{1}^{2}=x_{1}^{2} \cdot 1$.

Following the procedure of the "power transformation" [30], [29], we can obtain the dynamics of $\chi(x(t))=\chi(t)$, with which we will be able to compute the time derivative of the Lyapunov function. Namely, the dynamics will still be PWA, i.e. we will have

$$
\dot{\chi}(t)=\tilde{A}_{i} \chi(t) \text { for } \chi \in \tilde{X}_{i}, i \in \mathcal{I}
$$

with a new description of the cells $\tilde{X}_{i}$, thanks to matrices $\tilde{E}_{i} \in \mathbb{R}^{\tilde{l}_{i} \times \rho}$ such as

$$
\left\{\begin{array}{l}
x \in X_{i} \Rightarrow \chi \in \tilde{X}_{i} \\
\chi \in \tilde{X}_{i} \Rightarrow \tilde{E}_{i} \chi \geq 0
\end{array}\right.
$$

and a new definition for the matrices defining the boundaries, with matrices $\tilde{F}_{i j} \in \mathbb{R}^{\tilde{r}_{i j} \times \rho}$ such as

$$
\forall(i, j) \in \Gamma, \tilde{X}_{i} \cap \tilde{X}_{j} \subseteq\left\{\chi \mid \tilde{F}_{i j} \tilde{\chi}=0\right\}
$$

For the new system in (15), Assumptions 1, 2, 3 still hold:

1) sliding modes may occur, with dynamics:

$$
\dot{\chi}(t)=\left(\theta \tilde{A}_{i}+(1-\theta) \tilde{A}_{j}\right) \chi(t), 0 \leq \theta \leq 1
$$

2) there exists an equilibrium set $\tilde{E}_{e q} \mid \chi \in \tilde{E}_{e q} \Leftrightarrow \dot{\chi}=0$; $\chi \in \tilde{E}_{e q} \Leftrightarrow x \in E_{e q} ; \tilde{E}_{e q} \subset \bigcup_{i \in \mathcal{I}_{0}} \tilde{X}_{i}$

3) again we distinguish two cases:

- if the origin is in the interior of a cell $\tilde{X}_{i} ; \tilde{A}_{i}$ does not have any constant terms; $\tilde{\mathcal{A}}_{0}=\tilde{A}_{i}$;

- if the origin is in the boundary between cells $\tilde{X}_{i}$ and $\tilde{X}_{j}$ : there exist a $\tilde{\theta}, 0 \leq \tilde{\theta} \leq 1$, for which the constant terms disappear; we define $\tilde{\mathcal{A}}_{0}=\tilde{\theta} \tilde{A}_{i}+$ $(1-\tilde{\theta}) \tilde{A}_{j}$.

Moreover, $\tilde{\mathcal{A}}_{0}$ has $\tilde{h}$ eigenvectors (shared with $\tilde{A}_{i}$ and $\tilde{A}_{j}$ in the second case) that have 0 as associated eigenvalue. We call $\tilde{Z} \in \mathbb{R}^{\rho \times \tilde{h}}$ the full-rank matrix of these eigenvectors $\left(\tilde{\mathcal{A}}_{0} \tilde{Z}=\tilde{A}_{j} \tilde{Z}=\tilde{A}_{i} \tilde{Z}=0\right)$; $\tilde{\Pi} \in \mathbb{R}^{\rho \times(\rho-\tilde{h})}$ is the orthogonal complement of $\tilde{Z}$, with $\tilde{\Pi}^{T} \tilde{\Pi}=I$.

$V(\chi)$ in (10) is a candidate Lyapunov function for the system in (6). In order to ensure its continuity on the boundary between two cells $\tilde{X}_{i}$ and $\tilde{X}_{j},(i, j) \in \Gamma$, the following condition has to be satisfied:

$$
V_{i}(\chi)=V_{j}(\chi) \forall \chi(x) \in \tilde{X}_{i} \cap \tilde{X}_{j} .
$$

As a direct consequence of LaSalle's theorem ([18] page 128), the conditions that the candidate Lyapunov function must satisfy in order to prove the convergence to the equilibrium are in the following lemma.
Lemma 2: for the system in (6), the convergence of $x$ to $E_{e q}$ (which coincides with the convergence of $\chi$ in (15) to $\tilde{E}_{e q}$ ) for $t>0, t \rightarrow+\infty$ is assured under the following conditions:

1) the Lyapunov function is continuous, i.e. it satisfies (19);

2) the Lyapunov function is positive outside $\tilde{E}_{e q}$, i.e.

$$
V_{i}(\chi(x))>0 \text { for } \chi(x) \in \tilde{X}_{i} / \tilde{E}_{e q}, i \in \mathcal{I} \text {; }
$$

3) the derivative of the Lyapunov function is negative outside $\tilde{E}_{e q}$, i.e.

$$
\dot{V}_{i}(\chi(x))<0 \text { for } \chi(x) \in \tilde{X}_{i} / \tilde{E}_{e q}, i \in \mathcal{I}
$$

even in the case of sliding-mode dynamics;

4) the derivative of the Lyapunov function is null in $\tilde{E}_{e q}$, i.e.

$$
\dot{V}_{i}(\chi(x))=0 \text { for } \chi(x) \in \tilde{E}_{e q} \cap \tilde{X}_{i}, i \in \mathcal{I}_{0} .
$$

With this we are ready to introduce our main theoretical result.

\section{MAIN RESUlT}

Given the previous considerations and using the Sprocedure [3], we can arrive at the following theorem, which reveals that the Lyapunov function can be computed through an LMI optimization.

Theorem 3: Let us assume that the system in (6) satisfies Assumptions 1, 2 and 3. Let $\tilde{A}_{i}$ be the matrices describing the dynamics of the vector $\chi$ of degree $m$ deriving from $x$, as in (15), with $\tilde{X}_{i}, \tilde{E}_{i}, \tilde{F}_{i j}, \tilde{Z}, \tilde{\Pi}, \tilde{\mathcal{A}}_{0}$ as previously defined.

If there exist

- $P_{i}=P_{i}^{T} \in \mathbb{R}^{\rho \times \rho}$ for $i \in \mathcal{I}$;

- $U_{i}=U_{i}^{T}, W_{i}=W_{i}^{T} \in \mathbb{R}^{\tilde{l}_{i} \times \tilde{l}_{i}}$ and $\mathcal{T}_{i}, \mathcal{T}_{i}^{\prime}, \in \mathbb{R}^{\tilde{l}_{i}}$; all with non-negative entries for $i \in \mathcal{I}$;

- $U_{i j}=U_{i j}^{T}, U_{i j}^{\prime}=U_{i j}^{\prime T} \in \mathbb{R}^{\tilde{l}_{i} \times \tilde{l}_{i}}, \mathcal{T}_{i j}, \mathcal{T}_{i j}^{\prime} \in \mathbb{R}^{\tilde{l}_{i}}$, all with non-negative entries for $i, j \mid(i, j) \in \Gamma$;

- $L_{i j} \in \mathbb{R}^{\rho \times \tilde{r}_{i j}}$ for $i, j \mid(i, j) \in \Gamma$;

- $\tau_{i}(\nu), \tau_{i}^{\prime}(\nu), \tau_{i}^{\prime \prime}(\nu), \tau_{i j}(\nu), \tau_{i j}^{\prime}(\nu) \in \mathbb{R}$, for $i, j \in \mathcal{I}, \nu=$ $1, \ldots, \iota$

such that

$$
\begin{aligned}
P_{j}=P_{i}+\tilde{F}_{i j}^{T} L_{i j}^{T}+L_{i j} \tilde{F}_{i j}+H\left(\tau_{i j}\right) \text { for }(i, j) \in \Gamma \\
\qquad\left\{\begin{array}{l}
\tilde{\Pi}^{T} \tilde{A}_{i}^{T} P_{i} \tilde{Z}=0 \\
\tilde{E}_{i} \tilde{Z}=0
\end{array} \text { for } i \in \mathcal{I}_{0}\right.
\end{aligned}
$$

and the following LMIs hold

$$
\begin{gathered}
\left\{\begin{array}{l}
\tilde{\Pi}^{T}\left(P_{i}+H\left(\tau_{i}^{\prime}\right)-N_{i}\left(\mathcal{T}_{i}^{\prime}\right)-\tilde{E}_{i}^{T} W_{i} \tilde{E}_{i}\right) \tilde{\Pi} \succ 0 \\
\tilde{\Pi}^{T}\left(\tilde{A}_{i}^{T} P_{i}+P_{i} \tilde{A}_{i}+H\left(\tau_{i}^{\prime \prime}\right)+N_{i}\left(\mathcal{T}_{i}\right)+\tilde{E}_{i}^{T} U_{i} \tilde{E}_{i}\right) \tilde{\Pi} \prec 0 \\
\text { for } i \in \mathcal{I}_{0}
\end{array}\right. \\
\left\{\begin{array}{c}
P_{i}+H\left(\tau_{i}^{\prime}\right)-N_{i}\left(\mathcal{T}_{i}^{\prime}\right)-\tilde{E}_{i}^{T} W_{i} \tilde{E}_{i} \succ 0 \\
\tilde{A}_{i}^{T} P_{i}+P_{i} \tilde{A}_{i}+H\left(\tau_{i}^{\prime \prime}\right)+N_{i}\left(\mathcal{T}_{i}\right)+\tilde{E}_{i}^{T} U_{i} \tilde{E}_{i} \prec 0
\end{array}\right.
\end{gathered}
$$




$$
\begin{gathered}
\left\{\begin{array}{c}
\tilde{\Pi}^{T}\left(\tilde{A}_{i}^{T} P_{j}+P_{j} \tilde{A}_{i}+H\left(\tau_{i j}^{\prime}\right)+N_{i}\left(\mathcal{T}_{i j}\right)+N_{j}\left(\mathcal{T}_{i j}^{\prime}\right)\right. \\
\left.+\tilde{E}_{i}^{T} U_{i j} \tilde{E}_{i}+\tilde{E}_{j}^{T} U_{i j}^{\prime} \tilde{E}_{j}\right) \tilde{\Pi} \prec 0 \\
\tilde{\Pi}^{T}\left(\tilde{A}_{j}^{T} P_{i}+P_{i} \tilde{A}_{j}+H\left(\tau_{j i}^{\prime}\right)+N_{i}\left(\mathcal{T}_{j i}\right)+N_{j}\left(\mathcal{T}_{j i}^{\prime}\right)\right. \\
\left.+\tilde{E}_{j}^{T} U_{j i} \tilde{E}_{j}+\tilde{E}_{i}^{T} U_{j i}^{\prime} \tilde{E}_{i}\right) \tilde{\Pi} \prec 0
\end{array}\right. \\
\text { for }\left(i \in \mathcal{I}_{0}, j \in \mathcal{I}_{0}\right) \in \Gamma \\
\left\{\begin{array}{c}
\tilde{A}_{i}^{T} P_{j}+P_{j} \tilde{A}_{i}+H\left(\tau_{i j}^{\prime}\right)+N_{i}\left(\mathcal{T}_{i j}\right)+N_{j}\left(\mathcal{T}_{i j}^{\prime}\right) \\
+\tilde{E}_{i}^{T} U_{i j} \tilde{E}_{i}+\tilde{E}_{j}^{T} U_{i j}^{\prime} \tilde{E}_{j} \prec 0 \\
\tilde{A}_{j}^{T} P_{i}+P_{i} \tilde{A}_{j}+H\left(\tau_{j i}^{\prime}\right)+N_{i}\left(\mathcal{T}_{j i}\right)+N_{j}\left(\mathcal{T}_{j i}^{\prime}\right) \\
+\tilde{E}_{j}^{T} U_{j i} \tilde{E}_{j}+\tilde{E}_{i}^{T} U_{j i}^{\prime} \tilde{E}_{i} \prec 0 \\
\text { for }\left(i \notin \mathcal{I}_{0} \bigvee j \notin \mathcal{I}_{0}\right) \in \Gamma
\end{array}\right.
\end{gathered}
$$

with

$$
N_{i}(\mathcal{T})=\left[\begin{array}{c}
0 \\
\mathcal{T}^{T} \tilde{E}_{i}
\end{array}\right]+\left[\begin{array}{ll}
0 & \tilde{E}_{i}^{T} \mathcal{T}
\end{array}\right]
$$

and

$$
H(\tau)=\sum_{\nu=1}^{\iota} \tau(\nu) Q_{\nu}
$$

then the trajectories $x(t)$ of the system (6) converge asymptotically to the equilibrium set $E_{e q}$, with Lyapunov function (10).

Proof: consider the Lyapunov function candidate $V(\chi(x))$ defined by (10).

To prove (23) and (24), we use the same approach as in [12], [1]. So, for $\chi(x) \in \tilde{X}_{i} \cap \tilde{X}_{j}$, we have $\tilde{F}_{i j} \chi(x)=$ 0 ; replacing this into (23), multiplying on the right by $\chi(x)$ and on the left by $\chi(x)^{T}$, and remembering that $\chi(x)^{T} H(\tau) \chi(x)=0$ for any $\tau$, we get $\chi(x)^{T} P_{j} \chi(x)=$ $\chi(x)^{T} P_{i} \chi(x)$, that is (19), i.e. (23) implies continuity of the Lyapunov function on the boundaries.

Let us now consider (24) and (25). Based on Assumption 3, we can always write a decomposition for $\chi(x)$, of the kind $\chi(x)=\tilde{Z} \xi+\tilde{\Pi} \zeta$, with $\chi(x) \in \tilde{X}_{i}, i \in \mathcal{I}_{0}$. As $\tilde{\mathcal{A}}_{0} \tilde{Z}=0$ for $\chi(x) \in \tilde{E}_{e q}$ (i.e. $\chi(x)=\tilde{Z} \xi$ ), then the first in (24) implies (22). For $\chi(x) \notin \tilde{E}_{e q}$ instead, when the active dynamics is $\tilde{A}_{i}\left(i \in \mathcal{I}_{0}\right)$, we have $\dot{V}(\chi(x))=(\tilde{Z} \xi+$ $\tilde{\Pi} \zeta)^{T}\left(\tilde{A}_{i}^{T} P_{i}+P_{i} \tilde{A}_{i}\right)(\tilde{Z} \xi+\tilde{\Pi} \zeta)=\zeta^{T} \tilde{\Pi}^{T}\left(\tilde{A}_{i}^{T} P_{i}+P_{i} \tilde{A}_{i}^{T}\right) \tilde{\Pi} \zeta$ thanks to (24). Notice also that the second in (24) implies that $\tilde{E}_{i} \chi \geq 0 \Rightarrow \tilde{E}_{i} \tilde{\Pi} \zeta \geq 0$. Then, thanks to the S-procedure, the second in (25) implies $\dot{V}(\chi(x))<0$ for $\chi(x) \notin \tilde{E}_{e q}$, which is the first in (21), as we have $\zeta^{T} \tilde{\Pi}^{T} \tilde{E}_{i}^{T} U_{i} \tilde{E}_{i} \tilde{\Pi} \zeta \geq 0$ (true thanks to (16) and (24)), $\zeta^{T} \tilde{\Pi}^{T} N_{i}\left(\mathcal{T}_{i}^{\prime}\right) \Pi \zeta \geq 0$ (true thanks to (16) and (24)) and $\chi(x)^{T} H\left(\tau_{i}^{\prime}\right) \chi(x)=0$ (true thanks to (13)). In a similar way, the first expression in (25) naturally implies the first expression in (20) for the cells with $i \in \mathcal{I}_{0}$.

Subsequently, again thanks to the S-procedure, the first expression in (26) implies that $\chi(x)^{T} P_{i} \chi(x)>0$ when $\chi(x)^{T} \tilde{E}_{i}^{T} W_{i} \tilde{E}_{i} \chi(x) \geq 0$ (true thanks to (16) if $\left.\chi(x) \in \tilde{X}_{i}\right), \chi(x)^{T} N_{i}\left(\mathcal{T}_{i}^{\prime}\right) \chi(x) \geq 0$ (true thanks to (16)) $\chi(x)^{T} H\left(\tau_{i}^{\prime}\right) \chi(x)=0$ (true thanks to (13)), which in turn implies that $V_{i}(\chi(x))>0$ for $\chi(x) \in \tilde{X}_{i}$ i.e. the second expression in (20) for the cells with $i \in \mathcal{I}_{1}$. Similarly, the second expression in (26) ensures the second expression in (21).
The conditions (27) and (28) concern possible sliding modes on the boundary between two cells. Indeed, multiplying the second expression in (28) by $\theta$ and the second in (26) by $1-\theta$ and summing, we get (thanks to the S-procedure, as seen above)

$$
\chi(x)^{T}\left(\left(\theta \tilde{A}_{j}^{T}+(1-\theta) \tilde{A}_{i}^{T}\right) P_{i}+P_{i}\left(\theta \tilde{A}_{j}+(1-\theta) \tilde{A}_{i}\right)\right) \chi(x)<0
$$

for any $\chi \in \tilde{X}_{i} \cap \tilde{X}_{j}$, which ensures that the derivative of $V_{i}$ is negative for any possible sliding mode dynamics. Similarly, one can get the complementary expression

$$
\chi(x)^{T}\left(\left(\theta \tilde{A}_{i}^{T}+(1-\theta) \tilde{A}_{j}^{T}\right) P_{j}+P_{j}\left(\theta \tilde{A}_{i}+(1-\theta) \tilde{A}_{j}\right)\right) \chi(x)<0
$$

from the first in (28). Condition (27) concerns a sliding mode containing the equilibrium set, the inequalities can be proven with the same reasoning as for (28) and (25).

So we have shown that the conditions required by the theorem imply (19), (20), (21) and (22), satisfying the hypotheses of Lemma 2, which proves the theorem statement.

Remark 4: besides the linear matrix inequalities (LMIs) in (25), (26) and (28), Theorem 3 features some linear matrix equalities (LMEs) as well, (24) and (23). Such LMEs can be resolved by an appropriate parameterization of the involved matrices (for example, the most recent versions of Yalmip [20] can execute this function atomatically when introducing equality constraints).

Corollary 5: Theorem 1 in [1] is a special case of our Theorem 3, for the order of polynomial Lyapunov function $2(m=1)$ and without sliding modes on the boundaries between cells.

Remark 6: The condition (23) which ensures the continuity of the polynomial Lyapunov function, is adapted from the one developed in [1] which is equivalent to the one in [12]. So by this relation, all possible piecewise polynomial continuous functions are parameterized.

Remark 7: We can remark that conditions (28) which ensure the convergence of the sliding modes are less conservative than the ones imposed in [9] which requires the convex combination of the Lyapunov matrices.

Remark 8: The first conditions in (25) and (26) can be interpreted as a "sum of squares" property (SOS) [14], [23], [7], in fact we look for a positive definite polynomial $V_{i}(\chi)$ as a square product of a positive definite matrix times a vector of monomials $\chi$. The terms in $H(\tau)$ reduce the conservatism of the expressions.

\section{Application to the pneumatic actuator}

As shown in [1], and according to (3) and (4), the dynamical behavior of the pneumatic system described in Section II can then be cast into the form of a PWA system as in (6). Concerning the parameters of the test bench, we have $M=17 \mathrm{~kg}, F_{s}=40 \mathrm{~N}, S=7.27 \cdot 10^{-4} \mathrm{~m}^{2}$, $\varepsilon=0.1 \mathrm{~m} / \mathrm{s}, \varepsilon_{1}=0.005 \mathrm{~m}$ and $\varepsilon_{2}=0.01 \mathrm{~m} / \mathrm{s}$. Through a pole placement, according to the system specifications, we have set $k_{y}=50 \mathrm{~s}^{-3}, k_{v}=71 \mathrm{~s}^{-2}, k_{a}=51.4 \mathrm{~s}^{-1}$, $k_{P}=10 \mathrm{~s}^{-1}$ and $k_{N}=10 \mathrm{~s}^{-1}$. 
The switchings of the system (due to the friction model and to the control law) divide the state-space into a set of eight cells (see Fig. 4): two cells $X_{01}$ and $X_{02}$ including the origin ( 0 is on the boundary between these two cells), and six external cells $X_{1}, X_{1 s}, X_{2}, X_{2 s}, X_{3}$ and $X_{3 s}$, two by two symmetrical with respect to the origin.

The discontinuity of the friction model on $e_{v}=0$ can generate a sliding phenomenon on the corresponding boundary. So, to prove convergence of these sliding modes, we have to consider all cells associated to this boundary. For the stability analysis, whereas in the above properties, we consider only the following cells: $X_{01}, X_{02}, X_{1}, X_{2}$ and $X_{3}$. The conditions imposed on these cells will necessary be verified by symmetry for the other ones.

The piecewise polynomial function chosen in our case is of degree $2 m=4$. So, the power of monomials is $m=2$. We can construct the vector $\chi(x)$ according to (12), where $x$ in this case has the following form: $x=\left[x_{1}, x_{2}, x_{3}, x_{4}\right]^{T}$. So, $\chi(x)=\left[x_{1}, x_{2}, x_{3}, x_{4}, x_{1} x_{2}, x_{1} x_{3}, x_{1} x_{4}, x_{2} x_{3}, x_{2} x_{4}\right.$, $\left.x_{3} x_{4}, x_{1}^{2}, x_{2}^{2}, x_{3}^{2}, x_{4}^{2}, 1\right]^{T}$.

We can then construct the dynamics $\tilde{A}_{i}$ and the cells $\tilde{X}_{i}$. As we study the convergence of the state to $E_{e q}$, we consider a static setpoint, i.e. $y_{d}$ constant and $v_{d}=0, a_{d}=0$ (which implies $e_{v}=v, e_{a}=a$ ).

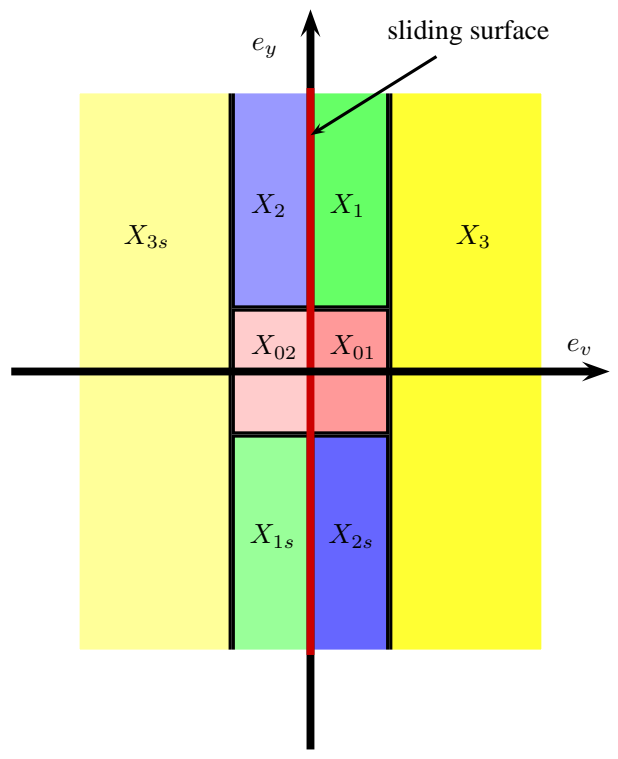

Fig. 4. The sections of the cells $X_{i}$ with respect to the $e_{y}$ and $e_{y}$ axes.

We analyse each cell in detail.

\section{- The central cell $X_{01}$}

In this cell the control law is $\# 2$, and the friction is $F_{f}(v)=+F_{s}$. The cell is active for

$$
\left\{\begin{array}{l}
\left|e_{y}\right| \leq \varepsilon_{1} \\
0 \leq v \leq \varepsilon_{2}
\end{array}\right.
$$

as $x \in X_{01} \Rightarrow \chi(x) \in \tilde{X}_{01}$, we define

$\tilde{E}_{01}=\left[\begin{array}{ccccccccccccccc}0 & 1 & 0 & 0 & 0 & 0 & 0 & 0 & 0 & 0 & 0 & 0 & 0 & 0 & 0 \\ 0 & -1 & 0 & 0 & 0 & 0 & 0 & 0 & 0 & 0 & 0 & 0 & 0 & 0 & \varepsilon_{2}\end{array}\right]$
Notice that this matrix $\tilde{E}_{01}$ describes only two of the four half-spaces defining the cell; the other two halfspace constraints (corresponding to two rows of the matrix) have been discarded in order to accommodate the condition in (24), i.e. $\tilde{E}_{01} \tilde{Z}=0$. Discarding lines in this way (which corresponds to having a bigger set for which the inequality $\tilde{E}_{01} \chi \geq 0$ holds than $\tilde{X}_{01}$ ) is allowed thanks to the definition of the matrices $\tilde{E}_{i}$, for which $\chi \in \tilde{X}_{i} \Rightarrow \tilde{E}_{i} \chi \geq 0$ but not viceversa, see (16). The matrix $\tilde{A}_{01}$ as well as those concerning the other cells are not shown due to space constraints.

- The central cell $X_{02}$

In this cell the control law is \#2, the friction is $F_{f}(v)=$ $-F_{s}$. The cell is active for

$$
\left\{\begin{array}{l}
\left|e_{y}\right| \leq \varepsilon_{1} \\
-\varepsilon_{2} \leq v \leq 0
\end{array}\right.
$$

for which we define

$\tilde{E}_{02}=\left[\begin{array}{ccccccccccccccc}0 & -1 & 0 & 0 & 0 & 0 & 0 & 0 & 0 & 0 & 0 & 0 & 0 & 0 & 0 \\ 0 & 1 & 0 & 0 & 0 & 0 & 0 & 0 & 0 & 0 & 0 & 0 & 0 & 0 & \varepsilon_{2}\end{array}\right]$

- The cell $X_{1}$

In this cell the control law is \#1, the friction is $F_{f}(v)=$ $+F_{s}$. The cell is active for

$$
\left\{\begin{array}{l}
e_{y}>\varepsilon_{1} \\
0 \leq v \leq \varepsilon_{2}
\end{array}\right.
$$

for which we define

$\tilde{E}_{1}=\left[\begin{array}{ccccccccccccccc}1 & 0 & 0 & 0 & 0 & 0 & 0 & 0 & 0 & 0 & 0 & 0 & 0 & 0 & -\varepsilon_{1} \\ 0 & -1 & 0 & 0 & 0 & 0 & 0 & 0 & 0 & 0 & 0 & 0 & 0 & 0 & \varepsilon_{2} \\ 0 & 1 & 0 & 0 & 0 & 0 & 0 & 0 & 0 & 0 & 0 & 0 & 0 & 0 & 0\end{array}\right]$

- The cell $X_{2}$

In this cell the control law is \#1, the friction is $F_{f}(v)=$ $-F_{s}$. The cell is active for

$$
\left\{\begin{array}{l}
e_{y}>\varepsilon_{1} \\
-\varepsilon_{2} \leq v \leq 0
\end{array}\right.
$$

for which we define

$$
\tilde{E}_{2}=\left[\begin{array}{ccccccccccccccc}
1 & 0 & 0 & 0 & 0 & 0 & 0 & 0 & 0 & 0 & 0 & 0 & 0 & 0 & -\varepsilon_{1} \\
0 & 1 & 0 & 0 & 0 & 0 & 0 & 0 & 0 & 0 & 0 & 0 & 0 & 0 & \varepsilon_{2} \\
0 & -1 & 0 & 0 & 0 & 0 & 0 & 0 & 0 & 0 & 0 & 0 & 0 & 0 & 0
\end{array}\right]
$$

- The cell $X_{3}$

In this cell the control law is \#1, the friction is $F_{f}(v)=$ $+F_{s}$. The cell is active for

$$
v>\varepsilon_{2}
$$

for which we define

$\tilde{E}_{3}=\left[\begin{array}{lllllllllllllll}0 & 1 & 0 & 0 & 0 & 0 & 0 & 0 & 0 & 0 & 0 & 0 & 0 & 0 & -\varepsilon_{2}\end{array}\right]$

- The cells $X_{1 s}, X_{2 s}$ and $X_{3 s}$ The conditions described above are also valid for the other cells $X_{1 s}, X_{2 s}$ and $X_{3 s}$, which are symmetrical with respect to the origin to the cells $X_{1}, X_{2}$ and $X_{3}$ respectively; just replace $\chi$ by $-\chi$.

Using Yalpim [20] and SeDuMi [26] under Matlab, Theorem 3 has been successfully applied by considering that sliding modes may occur on the boundary corresponding to $e_{v}=0$. The equilibrium is on the boundary between $X_{01}$ and 
$X_{02}$, for which we have $\tilde{\mathcal{A}}_{0}=\frac{1}{2} \tilde{A}_{01}+\frac{1}{2} \tilde{A}_{02}$. The level curves of the piecewise-polynomial Lyapunov function found have been plotted in Fig. 5. We can see that the level curves do not have a simple ellipsoidal shape; this implies that a simple common quadratic Lyapunov function (i.e., the same matrix $P$ for all the cells) is not sufficient to obtain such shapes. Moreover, we have verified that our test fails for the case of $m=1$, which justifies the need of a piecewise-polynomial function instead of a piecewise-quadratic one.

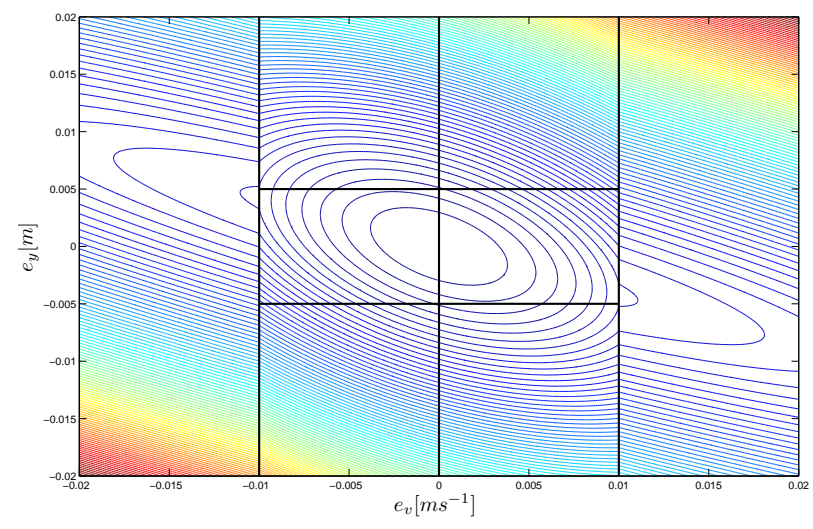

Fig. 5. Lyapunov function level curves on the $\left(e_{v}, e_{y}\right)$ plane.

\section{CONCLUSIONS}

In this paper, we present some sufficient conditions for finding piecewise-polynomial Lyapunov functions for PWA systems with sliding modes and equilibrium sets greater than the mere origin, and potentially on a sliding mode. We have applied this analysis to an electropneumatic system in closed-loop with a switching control law modeled as a PWA system with sliding modes due to presence of friction, for which our former work [1] was not applicable. The new extended conditions successfully found a 4-th degree piecewise-polynomial Lyapunov function through a convex optimization problem in terms of linear matrix inequalities.

The ideas in this paper can be extended to include the study of performance or to robust analysis, as a topic of future research.

\section{REFERENCES}

[1] O. Ameur, P. Massioni, G. Scorletti, X. Brun, and M. Smaoui. A piecewise-affine approach to the analysis of non-linear control laws for pneumatic systems. In Proc. of 53rd IEEE Conference on Decision and Control, December 2014.

[2] B. Armstrong-Hélouvry, P. Dupont, and C. Canudas De Wit. A survey of analysis tools and compensation methods for the control of machines with friction. Automatica, 30(7):1083-1138, July 1994.

[3] S. Boyd, L. El Ghaoui, E. Feron, and V. Balakrishnan. Linear Matrix Inequalities in System and Control Theory, volume 15 of SIAM Studies in Applied Mathematics. Society for Industrial and Applied Mathematics, 1994.

[4] M.S. Branicky. Multiple Lyapunov functions and other analysis tools for switched and hybrid systems. IEEE Transactions on Automatic Control, 43(4):475-482, April 1998.

[5] X. Brun, S. Sesmat, D. Thomasset, and S. Scavarda. Study of "sticking and restarting phenomenon" in electropneumatic positioning systems. Journal of Dynamic Systems Measurement and Control, 127(1):173184, May 2005.
[6] C. Canudas De Wit, H. Olsson, K.J. Åström, and P. Lischinsky. A new model for control of systems with friction. IEEE Transactions on Automatic Control, 40(3):419-425, March 1995.

[7] M.D. Choi, T.Y. Lam, and B. Reznick. Sums of squares of real polynomials. In Proceedings of Symposia in Pure mathematics, volume 58, pages 103-126. American Mathematical Society, 1995.

[8] P. R. Dahl. Measurement of solid friction parameters of ball bearings. Proceeding of 6th Annual Symposium on Incremental Motion Control System and Devices, pages 49-60, March 1977.

[9] T. Dezuo, L. Rodrigues, and A. Trofino. Stability analysis of piecewise affine systems with sliding modes. American Control Conference, pages 2005-2010, June 2014.

[10] A.F. Filippov and F.M. Arscott. Differential equations with discontinuous righthand sides: control systems, volume 18. Springer Science \& Business Media, 1988.

[11] L. Fridman, J. Moreno, and R. Iriarte. Sliding Modes After the First Decade of the 21st Century: State of the Art. Lecture Notes in Control and Information Sciences. Springer, 2011.

[12] A. Hassibi and S. Boyd. Quadratic stabilization and control of piecewise-linear systems. Proceedings of the American Control Conference, 6:3659-3664, Juin 1998.

[13] S. Hedlund and M. Johansson. A toolbox for computational analysis of piecewise linear systems. In Proceedings of European Control Conference, 1999.

[14] Z.W. Jarvis-Wloszek. Lyapunov based analysis and controller synthesis for polynomial systems using sum-of-squares optimization. $\mathrm{PhD}$ thesis, University of California, Berkeley, 2003.

[15] M. Johansson. Piecewise linear control systems. Springer, 2003.

[16] M. Johansson and A. Rantzer. Computation of piecewise quadratic Lyapunov functions for hybrid systems. IEEE Transaction in Automatic Control, 43(4):555-559, April 1998.

[17] D. Karnopp. Computer simulation of stick-slip friction in mechanical dynamic systems. Journal of Dynamic Systems, Measurement and Control, 107:100-103, March 1985.

[18] H.K. Khalil. Non Linear Systems, Third Edition. Prentice Hall, 2002.

[19] S. Krishnamurthy and J. Lee. A computational stability analysis of discrete-time piecewise linear systems. IEEE Conference on Decision and Control and 28th Chinese Control Conference, pages 1106-1111, December 2009.

[20] J. Löfberg. Yalmip: a toolbox for modeling and optimization in MATLAB. In Proc. of the CACSD Conference, pages 284-289, Taipei, Taiwan, 2004.

[21] M. Moarref and L. Rodrigues. Asymptotic stability of piecewise affine systems with sampled-data piecewise linear controllers. IEEE Conference on Decision and Control and European Control Conference (CDC-ECC), pages 8315-8320, December 2011.

[22] M.C. Oliveira and R. E. Skelton. Stability tests for constrained linear systems. In S.O.Reza Moheimani, editor, Perspectives in robust control, volume 268 of Lecture Notes in Control and Information Sciences, pages 241-257. Springer London, 2001.

[23] P.A. Parrilo. Semidefinite programming relaxations for semialgebraic problems. Mathematical programming, 96(2):293-320, May 2003.

[24] N.B.O.L. Pettit and E. P. Wellstead. Analyzing piecewise linear dynamical systems. IEEE Control Systems, 15(5):43-50, 1995.

[25] B. Samadi and L. Rodrigues. A unified dissipativity approach for stability analysis of piecewise smooth systems. Automatica, 47(12):27352742, December 2011.

[26] J.F. Sturm. Using SeDuMi 1.02, a MATLAB toolbox for optimization over symmetric cones. Optimization Methods and Software, 1112:625-653, 1999.

[27] K. Turki, M. Smaoui, D. Thomasset, and X. Brun. A solution to the "stick-slip" problem for an electropneumatic drive. International Journal of Fluid Power, 12(1):19-29, January 2011.

[28] A. Tustin. The effect of backlash and speed-dependent friction on the stability of closed-cycle control systems. Journal of the Institution of Electrical Engineers, 94(2A):143-151, January 1947.

[29] J. Xu and L. Xie. Homogeneous polynomial Lyapunov functions for piecewise affine systems. American Control Conference, 1:581-586, 2005.

[30] A.L. Zelentsovsky. Nonquadratic Lyapunov functions for robust stability analysis of linear uncertain systems. IEEE Transactions on Automatic Control, 39(1):135-138, January 1994. 